\title{
Article
}

\section{Convergence analysis for a fast class of multi-step Chebyshev-Halley-type methods under weak conditions}

\author{
Samundra Regmi ${ }^{1}$, Ioannis K. Argyros ${ }^{2, *}$ and Santhosh George ${ }^{3}$ \\ 1 Learning Commons, University of North Texas at Dallas, Dallas, TX, 75038, USA. \\ 2 Department of Mathematical Sciences, Cameron University, Lawton, OK 73505, USA. \\ 3 Department of Mathematical and Computational Sciences, National Institute of Technology Karnataka, India-575 025. \\ * Correspondence: iargyros@cameron.edu
}

Academic Editor: Krzysztof Gdawiec

Received: 27 November 2020; Accepted: 21 January 2021; Published: 29 January 2021.

\begin{abstract}
In this study a convergence analysis for a fast multi-step Chebyshe-Halley-type method for solving nonlinear equations involving Banach space valued operator is presented. We introduce a more precise convergence region containing the iterates leading to tighter Lipschitz constants and functions. This way advantages are obtained in both the local as well as the semi-local convergence case under the same computational cost such as: extended convergence domain, tighter error bounds on the distances involved and a more precise information on the location of the solution. The new technique can be used to extend the applicability of other iterative methods. The numerical examples further validate the theoretical results.
\end{abstract}

Keywords: Chebyshev method, Halley method, multi-step method, local, semi-local convergence, convergence order.

MSC: 65D10, 65G99, 65D99, 47J05, 49M15.

\section{Introduction}

I

$\mathrm{n}$ this study, we consider the problem of approximating a locally unique solution $x^{*}$ of the equation

$$
F(x)=0,
$$

where $F: \Omega \subseteq \mathcal{B}_{1} \longrightarrow \mathcal{B}_{2}$ be a Fréchet-differentiable operator, $\mathcal{B}_{1}$ and $\mathcal{B}_{2}$ are Banach spaces and $\Omega$ is a nonempty convex subset of $\mathcal{B}_{1}$. Numerous problems in Mathematics and computational sciences are written in the form of (1) using mathematical modeling [1-9]. Most solution methods for these equations are iterative, since closed form solutions can rarely be found. We study the local convergence of the multi-step Chebyshev-Halley-type [9] method defined for each $n=0,1,2, \ldots$ by

$$
\left\{\begin{array}{l}
y_{n}=x_{n}-\left[I+\frac{1}{2} K\left(x_{n}\right)\left(1-\delta K\left(x_{n}\right)\right)^{-1}\right] F\left(x_{n}\right)^{-1} F\left(x_{n}\right), \\
z_{n}=y_{n}-F^{\prime}\left(x_{n}\right)^{-1}\left[F^{\prime}\left(x_{n}\right)+F^{\prime \prime}\left(u_{n}\right)\left(x_{n}-v_{n}\right)\right] F^{\prime}\left(x_{n}\right)^{-1} F\left(y_{n}\right), \\
x_{n+1}=z_{n}-\left[I+K\left(x_{n}\right)+\gamma K\left(x_{n}\right)^{2}\right] F^{\prime}\left(x_{n}\right)^{-1} F\left(z_{n}\right),
\end{array}\right.
$$

where $x_{0}$ is an initial points, $\gamma, \delta \in S, S=\mathbb{R}$ or $S=\mathbb{C}, u_{n}=x_{n}-\frac{2}{3} F^{\prime}\left(x_{n}\right)^{-1} F\left(x_{n}\right), K\left(x_{n}\right)=$ $F^{\prime}\left(x_{n}\right)^{-1} F^{\prime \prime}\left(x_{n}\right) F^{\prime}\left(x_{n}\right)^{-1} F\left(x_{n}\right)$ and $v_{n}=x_{n}-F^{\prime}\left(x_{n}\right)^{-1} F\left(x_{n}\right)$.

The semi-local convergence of method (2) was given in [9] under the (C) conditions:

$\left(C_{1}\right)\left\|F^{\prime \prime}(x)\right\| \leq M$ for each $x \in \Omega$,

$\left(C_{2}\right) F^{\prime}\left(x_{0}\right)^{-1} \in \mathcal{L}\left(\mathcal{B}_{2}, \mathcal{B}_{1}\right)$ for some $x_{0} \in \Omega$ and $\left\|F^{\prime}\left(x_{0}\right)^{-1}\right\| \leq \beta$,

$\left.\left(C_{3}\right) \| F^{\prime}\left(x_{0}\right)^{-1} F 9 x_{0}\right) \| \leq \eta$

and

$\left(C_{4}\right)\left\|F^{\prime \prime}(x)-F^{\prime \prime}(y)\right\| \leq \varphi(\|x-y\|)$ for each $x, y \in \Omega$ and $\varphi$ satisfies $\varphi(0) \geq 0, \varphi(t s) \leq t^{a} \varphi(s)$ for each $t \in[0,1], s \in(0,+\infty), 0 \leq a \leq 1$.

Similar methods have been consider by other authors using conditions $\left(C_{1}\right)-\left(C_{4}\right)$ but the convergence order is smaller [4-8]. The convergence order was shown to be $4+3 a$ using recurrence relations [9]. Notice 
that method (2) uses the second Fréchet-derivative which is expensive in general. However, there are cases e.g., when $F^{\prime \prime}$ is a bilinear operator or other cases $[1-3,7,8]$, when method (2) is very use full, since it is very fast. Condition $\left(C_{4}\right)$ may be hard to verify or may not hold even in simple cases. Let us consider $\varphi(t)=b t$ for some $b>0$. Consider a motivational example. Define function $F$ on $\Omega=\left[-\frac{1}{2}, \frac{5}{2}\right]$ by

$$
F(x)=\left\{\begin{array}{l}
x^{2} \ln x^{2}+2 x^{4}-4 x^{3}+2 x^{2}, x \neq 0, \\
0, x=0 .
\end{array}\right.
$$

Choose $x^{*}=1$. Then, condition $\left(C_{4}\right)$ is not satisfied since the third Fréchet derivative does not exists at $x^{*}=1$.

In this study, we present the local convergence not given in [9] and drop condition $\left(C_{4}\right)$. This way we expand the applicability of method (2). Moreover, we refine Theorem $1 \mathrm{in}$ [9] leading to a new semi-local convergence for method (2), a wider convergence region, tighter error bounds on the distances $\left\|x_{n}-x^{*}\right\|$ and an at least as precise information on the location of the solution. These advantages are obtained, since we find a more precise region where the iterate lie resulting to tighter Lipschitz constants as well as the aforementioned advantages. The new constants are special cases of the old ones, so the advantages are obtained under the same computational cost.

The study is structured as follows; Section 2 contain the local convergence followed by the semi-local convergence in Section 3. The numerical examples in Section 4 conclude this study.

\section{Local convergence}

The local convergence that follows is centered on some parameters and scalar functions. Let $\gamma, \delta \in \mathbb{R}, w_{0}$ : $[0,+\infty) \longrightarrow[0,+\infty)$ be a continuous and non-decreasing function with $w_{0}(0)=0$. Define parameter $\rho_{0}$ by

$$
\rho_{0}=\sup \left\{t \geq 0: w_{0}(t)<1\right\} .
$$

Let $v_{1}, v:\left[0, \rho_{0}\right) \longrightarrow[0,+\infty)$ be continuous, non-decreasing functions and $\delta \in \mathbb{R}$. Define functions $g_{0}$ and $h_{0}$ on the interval $\left[0, \rho_{0}\right)$ by

$$
g_{0}(t)=\frac{v_{1}(t) \int_{0}^{1} v(\theta t) d \theta}{1-w_{0}(t)}
$$

and

$$
h_{0}(t)=|\delta| g_{0}(t) t-1
$$

Suppose

$$
h_{0}(t) \longrightarrow+\infty \text { or a positive constant as } t \longrightarrow \rho_{0}^{-},
$$

we have $h_{0}(0)=-1$. It follows by the intermediate value theorem that equation $h_{0}(t)=0$ has solutions in $\left(0, \rho_{0}\right)$. Denote by $\rho$ the smallest such solution in $\left(0, \rho_{0}\right)$. Define functions $g_{1}$ and $h_{1}$ on $[0, \rho)$ by

$$
g_{1}(t)=\frac{\int_{0}^{1} w((1-\theta) t) d \theta}{1-w_{0}(t)}+\frac{g_{0}(t) t \int_{0}^{1} v(\theta t) d \theta}{2\left(1-|\delta| g_{0}(t) t\right)\left(1-w_{0}(t)\right)}
$$

and

$$
h_{1}(t)=g_{1}(t)-1,
$$

where $w:[0, \rho) \longrightarrow[0,+\infty)$ is a continuous and non-decreasing function with $w(0)=0$.

Suppose

$$
h_{1}(t) \longrightarrow+\infty \text { or a positive constant as } t \longrightarrow \rho^{-},
$$

we get $h_{1}(0)=-1$. It follows by the intermediate value theorem that equation $h_{1}(t)=0$ has solutions in $(0, \rho)$. Denote by $r_{1}$ the smallest such solution in $(0, \rho)$. Suppose that

$$
v(0)<3 .
$$

Define functions $p$ and $q$ on the interval $\left[0, \rho_{0}\right)$ by

$$
p(t)=\frac{\int_{0}^{1} w((1-\theta) t) d \theta+\frac{1}{3} \int_{0}^{1} v(\theta t) d \theta}{1-w_{0}(t)}
$$


and

$$
q(t)=p(t) t-1
$$

Suppose

$$
q(t) \longrightarrow+\infty \text { or a positive constant as } t \longrightarrow \rho_{0}^{-},
$$

we obtain that $q(0)=\frac{v(0)}{3}-1<0$. Denote by $r_{q}$ the smallest solution of equation $q(t)=0$ in $\left(0, \rho_{0}\right)$. Define functions $g_{2}$ and $h_{2}$ on the interval $[0, \rho)$ by

$$
\begin{aligned}
g_{2}(t)= & \frac{\int_{0}^{1} w\left((1-\theta) g_{1}(t) t\right) d \theta g_{1}(t)}{1-w_{0}\left(g_{1}(t) t\right)}+\frac{\left(w_{0}(t)+w_{0}\left(g_{1}(t) t\right)\right) \int_{0}^{1} v\left(\theta g_{1}(t) t\right) d \theta g_{1}(t)}{\left(1-w_{0}(t)\right)\left(1-w_{0}\left(g_{1}(t) t\right)\right)} \\
& +\frac{\bar{v}(p(t) t) \int_{)}^{1} v(\theta t) d \theta \int_{0}^{1} v\left(\theta g_{1}(t) t\right) g_{1}(t)}{\left(1-w_{0}(t)\right)^{3}}
\end{aligned}
$$

and

$$
h_{2}(t)=g_{2}(t)-1 \text {, }
$$

where function $\bar{v}:\left[0, \rho_{0}\right) \longrightarrow[0,+\infty)$ is continuous and nondecreasing. Define parameter $\bar{\rho}_{0}$ by

$$
\bar{\rho}_{0}=\max \left\{t \in\left[0, \rho_{0}\right]: w_{0}\left(g_{1}(t) t\right)<1\right\} .
$$

\section{Suppose}

$$
h_{2}(t) \longrightarrow+\infty \text { or a positive constant as } t \longrightarrow \bar{\rho}_{0}^{-},
$$

we have $h_{2}(0)=-1<0$. Denote by $r_{2}$ the smallest solution of equation $h_{2}(t)=0$ in $\left(0, \bar{\rho}_{0}\right)$. Define parameter $\overline{\bar{\rho}}_{0}$ by

$$
\overline{\bar{\rho}}_{0}=\max \left\{t \in\left[0, \rho_{0}\right]: w_{0}\left(g_{2}(t) t\right)<1\right\} .
$$

Define functions $g_{3}$ and $h_{3}$ on the interval $[0, \lambda), \lambda=\min \left\{\bar{\rho}_{0}, \overline{\bar{\rho}}_{0}\right\}$ by

$$
\begin{aligned}
g_{3}(t)= & \frac{\int_{0}^{1} w\left((1-\theta) g_{2}(t) t\right) d \theta g_{2}(t)}{1-w_{0}\left(g_{2}(t) t\right)}+\frac{\left(w_{0}(t)+w_{0}\left(g_{2}(t) t\right)\right) \int_{0}^{1} v\left(\theta g_{2}(t) t\right) d \theta g_{2}(t)}{\left(1-w_{0}(t)\right)\left(1-w_{0}\left(g_{2}(t) t\right)\right)} \\
& +\frac{g_{0}(t) \int_{0}^{1} v\left(\theta g_{2}(t) t\right) d \theta g_{2}(t) t}{1-w_{0}(t)}+\frac{|\gamma| g_{0}^{2}(t) \int_{0}^{1} v\left(\theta g_{2}(t) t\right) d \theta g_{2}(t) t^{2}}{1-w_{0}(t)}
\end{aligned}
$$

and

$$
h_{3}(t)=g_{3}(t)-1
$$

Suppose

$$
h_{3}(t) \longrightarrow+\infty \text { or a positive constant as } t \longrightarrow \lambda^{-},
$$

we get that $h_{3}(0)=-1$. Denote by $r_{3}$ the smallest solution of equation $h_{3}(t)=0$ in $(0, \lambda)$. Define the radius of convergence $r$ by

$$
r=\min \left\{r_{i}\right\}, i=1,2,3 \text {. }
$$

Then for each $t \in[0, r)$

$$
\begin{aligned}
& 0 \leq g_{i}(t)<1, \\
& 0 \leq w_{0}(t)<1, \\
& 0 \leq w_{0}\left(g_{1}(t) t\right)<1, \\
& 0 \leq w_{0}\left(g_{2}(t) t\right)<1,
\end{aligned}
$$

and

$$
0 \leq p(t) t<1 .
$$

Some alternatives to the aforementioned conditions are; Equation

$$
w_{0}(t)=1
$$


has positive solutions. Denoted by $\rho_{0}$ the smallest such solution. Similarly, define $\bar{\rho}_{0}, \overline{\bar{\rho}}_{0}$. Functions, $v_{1}, v, w, \bar{v}$ defined on the same intervals as before are continuous and increasing. Then, clearly conditions (4), (5), (7), (8) and (9) hold.

Let $U(x, \mu), \bar{U}(x, \mu)$ denote the open and closed balls in $\mathcal{B}_{1}$, respectively of center $x \in \mathcal{B}_{1}$ and of radius $\mu>0$. The local convergence of method (2) is based on the preceding notation and conditions $(A)$

$\left(a_{1}\right) F: \Omega \subset \mathcal{B}_{1} \longrightarrow \mathcal{B}_{2}$ is a twice continuously Fréchet differentiable operator.

$\left(a_{2}\right)$ There exists $x^{*} \in \Omega$ such that $F\left(x^{*}\right)=0$ and $F^{\prime}\left(x^{*}\right)^{-1} \in \mathcal{L}\left(\mathcal{B}_{1}, \mathcal{B}_{1}\right)$.

$\left(a_{3}\right)$ There exists function $w_{0}:[0,+\infty) \longrightarrow[0,+\infty)$ continuous and non-decreasing with $w_{0}(0)=0$ such that for each $x \in \Omega$

$$
\left\|F^{\prime}\left(x^{*}\right)^{-1}\left(F^{\prime}(x)-F^{\prime}\left(x^{*}\right)\right)\right\| \leq w_{0}\left(\left\|x-x^{*}\right\|\right) .
$$

Set $\Omega_{0}=\Omega \cap U\left(x^{*}, \rho_{0}\right)$, where $\rho_{0}$ is defined by (3).

$\left(a_{4}\right)$ There exist functions $w:\left[0, \rho_{0}\right) \longrightarrow[0,+\infty), v:\left[0, \rho_{0}\right) \longrightarrow[0,+\infty), \bar{v}:\left[0, \rho_{0}\right) \longrightarrow[0,+\infty)$ continuous and nondecreasing with $w(0)=0$ such that for each $x, y \in \Omega_{0}$

$$
\begin{gathered}
\left\|F^{\prime}\left(x^{*}\right)^{-1}\left(F^{\prime}(x)-F^{\prime}(y)\right)\right\| \leq w(\|x-y\|), \\
\left\|F^{\prime}\left(x^{*}\right)^{-1} F^{\prime}(x)\right\| \leq v\left(\left\|x-x^{*}\right\|\right)
\end{gathered}
$$

and

$$
\left\|F^{\prime}\left(x^{*}\right)^{-1} F^{\prime \prime}(x)\right\| \leq \bar{v}\left(\left\|x-x^{*}\right\|\right) .
$$

$\left(a_{5}\right) \bar{U}\left(x^{*}, r\right) \subseteq D$ and (6) hold, where $r$ is given by (10).

$\left(a_{6}\right)$ There exists $R \geq r$ such that

$$
\int_{0}^{1} w_{0}(\theta R) d \theta<1
$$

Theorem 1. Suppose that the conditions (A) and (4)-(9) hold. Then, sequence $\left\{x_{n}\right\}$ generated for $x_{0} \in U\left(x^{*}, r\right)-\left\{x^{*}\right\}$ by method (2) is well defined in $U\left(x^{*}, r\right)$ remains in $U\left(x^{*}, r\right)$ for each $n=0,1,2, \ldots$ and converges to $x^{*}$. Moreover, the following estimates hold

$$
\begin{gathered}
\left\|y_{n}-x^{*}\right\| \leq g_{1}\left(\left\|x_{n}-x^{*}\right\|\right)\left\|x_{n}-x^{*}\right\| \leq\left\|x_{n}-x^{*}\right\|<r, \\
\left\|z_{n}-x^{*}\right\| \leq g_{2}\left(\left\|x_{N}-x^{*}\right\|\right)\left\|x_{n}-x^{*}\right\| \leq\left\|x_{n}-x^{*}\right\|,
\end{gathered}
$$

and

$$
\left\|x_{n+1}-x^{*}\right\| \leq g_{3}\left(\left\|x_{n}-x^{*}\right\|\right)\left\|x_{n}-x^{*}\right\| \leq\left\|x_{n}-x^{*}\right\|,
$$

where the functions $g_{i}, i=1,2,3$ are defined previously. Furthermore, the point $x^{*}$ is the only solution of equation $F(x)=0$ in $\Omega_{1}=\Omega \cap \bar{U}\left(x^{*}, R\right)$.

Proof. Mathematical induction is employed to show estimates (16)-(18). Using (3), $\left(a_{1}\right)-\left(a_{3}\right)$ and $x_{0} \in U\left(x^{*}, r\right)$, we have that

$$
\left\|F^{\prime}\left(x^{*}\right)^{-1}\left(F^{\prime}(x)-F^{\prime}\left(x^{*}\right)\right)\right\| \leq w_{0}\left(\left\|x_{0}-x^{*}\right\|\right) \leq w_{0}(r)<1 .
$$

In view of (19) and the Banach lemma on invertible operators $[1-3,7,8]$, we get that $F^{\prime}\left(x_{0}\right)^{-1} \in \mathcal{L}\left(\mathcal{B}_{2}, \mathcal{B}_{1}\right)$,

$$
\left\|F^{\prime}(x)^{-1} F^{\prime}\left(x^{*}\right)\right\| \leq \frac{1}{\left.1-w_{0}(\| x)-x^{*} \|\right)^{\prime}}
$$

and $u_{0}$ is well defined. By the definition of operator $K,\left(a_{4}\right)$ and (20), we obtain that

$$
\begin{aligned}
& \left\|K\left(x_{0}\right)\right\| \leq\left\|F^{\prime}\left(x_{0}\right)^{-1} F^{\prime}\left(x^{*}\right)\right\|\left\|F^{\prime}\left(x^{*}\right)^{-1} F^{\prime \prime}\left(x_{0}\right)\right\|\left\|F^{\prime}\left(x_{0}\right)^{-1} F^{\prime}\left(x^{*}\right)\right\|\left\|F^{\prime}\left(x^{*}\right)^{-1} F\left(x_{0}\right)\right\| \\
& \leq \frac{\bar{v}\left(\left\|x_{0}-x^{*}\right\|\right) \int_{0}^{1} v\left(\theta\left\|x_{0}-x^{*}\right\|\right) d \theta\left\|x_{0}-x^{*}\right\|}{\left(1-w_{0}\left(\left\|x_{0}-x^{*}\right\|\right)\right)^{2}} \\
& \leq g_{0}\left(\left\|x_{0}-x^{*}\right\|\right)\left\|x_{0}-x^{*}\right\|, \\
& \left\|\delta K\left(x_{0}\right)\right\| \leq|\delta| g_{0}\left(\left\|x_{0}-x^{*}\right\|\right)\left\|x_{0}-x^{*}\right\| \\
& \leq|\delta| g_{0}(r)<1 \text {, }
\end{aligned}
$$


so $\delta K\left(x_{0}\right)^{-1} \in \mathcal{L}\left(\mathcal{B}_{2}, \mathcal{B}_{1}\right)$,

$$
\left\|\left(I-\delta K\left(x_{0}\right)\right)^{-1}\right\| \leq \frac{1}{1-|\delta| g_{0}\left(\left\|x_{0}-x^{*}\right\|\right)\left\|x_{0}-x^{*}\right\|}
$$

and

$$
\left\|K\left(x_{0}\right)\left(I-\delta K\left(x_{0}\right)^{-1}\right)\right\| \leq \frac{g_{0}\left(\left\|x_{0}-x^{*}\right\|\right)\left\|x_{0}-x^{*}\right\|}{1-|\delta| g_{0}\left(\left\|x_{0}-x^{*}\right\|\right)\left\|x_{0}-x^{*}\right\|} .
$$

By the first substep of method (2), we can write

$$
y_{0}-x^{*}=x_{0}-x^{*}-F^{\prime}\left(x_{0}\right)^{-1} F\left(x_{0}\right)-\frac{1}{2} K\left(x_{0}\right)\left(1-\delta K\left(x_{0}\right)\right)^{-1} F^{\prime}\left(x_{0}\right)^{-1} F\left(x_{0}\right) .
$$

Then, using (10), (11) (for $i=1),\left(a_{4}\right),(21)-(25)$, we have in turn that

$$
\begin{aligned}
\left\|y_{0}-x^{*}\right\| \leq & \left\|F^{\prime}\left(x_{0}\right)^{-1} F^{\prime}\left(x^{*}\right)\right\| \int_{0}^{1}\left\|F^{\prime}\left(x^{*}\right)^{-1}\left(F^{\prime}\left(x^{*}+\theta\left(x_{0}-x^{*}\right)\right)-F^{\prime}\left(x_{0}\right)\right)\left(x_{0}-x^{*}\right) d \theta\right\| \\
& +\frac{1}{2}\left\|K\left(x_{0}\right)\left(1-\delta K\left(x_{0}\right)\right)^{-1}\right\| \\
\leq & g_{1}\left(\left\|x_{0}-x^{*}\right\|\right)\left\|x_{0}-x^{*}\right\| \leq\left\|x_{0}-x^{*}\right\|<r
\end{aligned}
$$

which shows (16) for $n=0$ and $y_{0} \in U\left(x^{*}, r\right)$. The point $v_{0} \in U\left(x^{*}, r\right)$ by (26) and the choice of $r$. We shall show that $u_{0} \in U\left(x^{*}, r\right)$. By the definition of $u_{0}$, we get in turn that

$$
\begin{aligned}
\left\|u_{0}-x^{*}\right\| & =\left\|\left(x_{0}-x^{*}-F^{\prime}\left(x_{0}\right)^{-1} F\left(x_{0}\right)\right)+\frac{1}{3} F^{\prime}\left(x_{0}\right)^{-1} F\left(x_{0}\right)\right\| \\
& \leq \frac{\int_{0}^{1} w\left((1-\theta)\left\|x_{0}-x^{*}\right\|\right) d \theta\left\|x_{0}-x^{*}\right\|}{1-w_{0}\left(\left\|x_{0}-x^{*}\right\|\right)}+\frac{1}{3} \frac{\int_{0}^{1} v\left(\theta\left\|x_{0}-x^{*}\right\|\right) d \theta\left\|x_{0}-x^{*}\right\|}{1-w_{0}\left(\left\|x_{0}-x^{*}\right\|\right)} \\
& \leq p\left(\left\|x_{0}-x^{*}\right\|\right)\left\|x_{0}-x^{*}\right\| \leq\left\|x_{0}-x^{*}\right\|<r
\end{aligned}
$$

so $u_{0} \in U\left(x^{*}, r\right)$. We can write by the second substep of method (2)

$$
\begin{aligned}
z_{0}-x^{*}= & y_{0}-x^{*}-F^{\prime}\left(x_{0}\right)^{-1} F\left(y_{0}\right)-F^{\prime}\left(x_{0}\right)^{-1} F^{\prime \prime}\left(u_{0}\right)\left(x_{0}-y_{0}\right) F^{\prime}\left(x_{0}\right)^{-1} F\left(y_{0}\right) \\
= & \left(y_{0}-x^{*}-F^{\prime}\left(y_{0}\right)^{-1} F\left(y_{0}\right)\right)+F^{\prime}\left(y_{0}\right)^{-1}\left(F^{\prime}\left(x_{0}\right)-F^{\prime}\left(y_{0}\right)\right) F^{\prime}\left(x_{0}\right)^{-1} F\left(y_{0}\right) \\
& -F^{\prime}\left(x_{0}\right)^{-1} F^{\prime \prime}\left(u_{0}\right)\left(x_{0}-v_{0}\right) F^{\prime}\left(x_{0}\right)^{-1} F\left(y_{0}\right)
\end{aligned}
$$

so

$$
\begin{aligned}
\left\|z_{0}-x^{*}\right\|= & \frac{\int_{0}^{1} w\left((1-\theta)\left\|y_{0}-x^{*}\right\|\right) d \theta\left\|y_{0}-x^{*}\right\|}{1-w_{0}\left(\left\|y_{0}-x^{*}\right\|\right)} \\
& +\frac{\left(w_{0}\left(\left\|x_{0}-x^{*}\right\|\right)+w_{0}\left(\left\|y_{0}-x^{*}\right\|\right)\right) \int_{0}^{1} v\left(\theta\left\|y_{0}-x^{*}\right\|\right) d \theta\left\|y_{0}-x^{*}\right\|}{\left(1-w_{0}\left(\left\|y_{0}-x^{*}\right\|\right)\right)\left(1-w_{0}\left(\left\|x_{0}-x^{*}\right\|\right)\right)} \\
& +\bar{v}\left(p\left(\left\|x_{0}-x^{*}\right\|\right)\left\|x_{0}-x^{*}\right\|\right) \frac{\int_{0}^{1} v\left(\theta\left\|y_{0}-x^{*}\right\|\right) d \theta\left\|x_{0}-x^{*}\right\| \int_{0}^{1} v\left(\theta\left\|y_{0}-x^{*}\right\|\right) d \theta\left\|y_{0}-x^{*}\right\|}{\left(1-w_{0}\left(\left\|x_{0}-x^{*}\right\|\right)\right)^{2}} \\
\leq & g_{2}\left(\left\|x_{0}-x^{*}\right\|\right)\left\|x_{0}-x^{*}\right\| \leq\left\|x_{0}-x^{*}\right\|<r
\end{aligned}
$$

so (17) holds for $n=0$ and $z_{0} \in U\left(x^{*}, r\right)$. Then, by the third substep of method (2) we can also write.

$$
\begin{aligned}
x_{1}-x_{0}= & z_{0}-x^{*}-F^{\prime}\left(z_{0}\right)^{-1} F\left(z_{0}\right)+F^{\prime}\left(z_{0}\right)^{-1}\left(F^{\prime}\left(x_{0}\right)-F^{\prime}\left(z_{0}\right)\right) F^{\prime}\left(x_{0}\right)^{-1} F\left(z_{0}\right) \\
& -K\left(x_{0}\right) F^{\prime}\left(x_{0}\right)^{-1} F\left(z_{0}\right)-\gamma K\left(x_{0}\right)^{2} F^{\prime}\left(x_{0}\right)^{-1} F\left(z_{0}\right),
\end{aligned}
$$

so

$$
\begin{aligned}
\left\|x_{1}-x^{*}\right\| \leq & \frac{\int_{0}^{1} w\left((1-\theta)\left\|z_{0}-x^{*}\right\|\right) d \theta\left\|z_{0}-x^{*}\right\|}{1-w_{0}\left(\left\|z_{0}-x^{*}\right\|\right)} \\
& +\frac{\left(w_{0}\left(\left\|x_{0}-x^{*}\right\|\right)+w_{0}\left(\left\|z_{0}-x^{*}\right\|\right)\right) \int_{0}^{1} v\left(\theta\left\|z_{0}-x^{*}\right\|\right) d \theta\left\|z_{0}-x^{*}\right\|}{\left(1-w_{0}\left(\left\|z_{0}-x^{*}\right\|\right)\right)\left(1-w_{0}\left(\left\|x_{0}-x^{*}\right\|\right)\right)}
\end{aligned}
$$




$$
\begin{aligned}
& +\frac{g_{0}\left(\left\|x_{0}-x^{*}\right\|\right)\left\|x_{0}-x^{*}\right\| \int_{0}^{1} v\left(\theta\left\|z_{0}-x^{*}\right\|\right) d \theta\left\|z_{0}-x^{*}\right\|}{\left(1-w_{0}\left(\left\|x_{0}-x^{*}\right\|\right)\right)} \\
& +|\gamma| \frac{g_{0}^{2}\left(\left\|x_{0}-x^{*}\right\|\right)\left\|x_{0}-x^{*}\right\|^{2} \int_{0}^{1} v\left(\theta\left\|z_{0}-x^{*}\right\|\right) d \theta\left\|z_{0}-x^{*}\right\|}{\left(1-w_{0}\left(\left\|x_{0}-x^{*}\right\|\right)\right)} \\
\leq & \left.g_{3}(\| x)-x^{*} \|\right)\left\|x_{0}-x^{*}\right\| \leq\left\|x_{0}-x^{*}\right\|<r,
\end{aligned}
$$

so (18) holds for $n=0$ and $x_{1} \in U\left(x^{*}, r\right)$. The induction for (16)-(18) is completed, if we simply replace $x_{0}, y_{0}, z_{0}, u_{0}, v_{0}, x_{1}$ by $x_{m}, y_{m}, z_{m} . u_{m}, v_{m}, x_{m+1}$ in the preceding estimates. Then, from the estimate

$$
\left\|x_{m+1}-x^{*}\right\| \leq c\left\|x_{m}-x^{*}\right\|<r, c=g_{3}\left(\left\|x_{0}-x^{*}\right\|\right) \in[0,1)
$$

we conclude that $\lim _{m \longrightarrow \infty} x_{m}=x^{*}$ and $x_{m+1} \in U\left(x^{*}, r\right)$. Let $y^{*} \in \Omega_{1}$ with $F\left(y^{*}\right)=0$. Define linear operator $T=\int_{0}^{1} F^{\prime}\left(x^{*}+\theta\left(y^{*}-x^{*}\right)\right) d \theta$. Then, using $\left(a_{3}\right)$ and $\left(a_{6}\right)$, we get that

$$
\left\|F^{\prime}\left(x^{*}\right)^{-1}\left(T-F^{\prime}\left(x^{*}\right)\right)\right\| \leq \int_{0}^{1} w_{0}\left(\theta\left\|y^{*}-x^{*}\right\|\right) d \theta \leq \int_{0}^{1} w_{0}(\theta R) d \theta<1,
$$

so $T^{-1} \in \mathcal{L}\left(\mathcal{B}_{2}, \mathcal{B}_{1}\right)$. Using the identity $0=F\left(y^{*}\right)-F\left(x^{*}\right)=T\left(y^{*}-x^{*}\right)$, we deduce that $x^{*}=y^{*}$, which completes the uniqueness part of the proof.

Remark 1. (a) Let $w_{0}(t)=L_{0} t, w(t)=L t$ and $w^{*}(t)=L^{*} t \quad\left(w^{*}\right.$ replacing $w$ in $\left.\left(a_{4}\right)\right)$. In [9], authors used instead of $\left(a_{4}\right)$ the condition

$$
\left\|F^{\prime}\left(x_{0}\right)^{-1}\left(F^{\prime}(x)-F^{\prime}(y)\right)\right\| \leq L^{*}\|x-y\| \text { for each } x, y \in \Omega .
$$

But using $\left(a_{4}\right)$ and (33) we get that

$$
L \leq L^{*}
$$

holds, since $\Omega_{0} \subseteq \Omega$. In case $L<L^{*}$. Then, the new convergence analysis is better than the old one. Notice also that we have by $\left(a_{3}\right)$ and (33)

$$
L_{0} \leq L^{*}
$$

The advantages are obtained under the same computational cost as before, since in practice the computation of constant $L^{*}$ requires the computation of $L_{0}$ and $L$ as special cases. In the literature (with the exception of our works) (33) is only used for the computation of the upper bounds of the inverses of the operators involved.

(b) The radius $r_{A}=\frac{2}{2 L_{0}+L}$ was obtained in [1-3] as the convergence radius for Newton's method under condition $\left(a_{1}\right)-\left(a_{4}\right)$. Notice that the convergence radius for Newton's method given independently by Rheinboldt (see [1]) and Traub (see [2]) is given by

$$
\rho=\frac{2}{3 L^{*}}<r_{A} .
$$

As an example, let us consider the function $f(x)=e^{x}-1$. Then $x^{*}=0$. Set $\Omega=U(0,1)$. Then, we have that $L_{0}=e-1<L^{*}=e, L=e^{\frac{1}{e-1}}$, so $\rho=0.24252961<r_{A}=0.3827$.

Moreover, the new error bounds [1-3] are

$$
\left\|x_{n+1}-x^{*}\right\| \leq \frac{L}{1-L_{0}\left\|x_{n}-x^{*}\right\|}\left\|x_{n}-x^{*}\right\|^{2},
$$

whereas the old ones $[7,8]$

$$
\left\|x_{n+1}-x^{*}\right\| \leq \frac{L}{1-L\left\|x_{n}-x^{*}\right\|}\left\|x_{n}-x^{*}\right\|^{2} .
$$

Clearly, the new error bounds are more precise, if $L_{0}<L$. Moreover, the radius of convergence of method (2) given by $r$ is smaller than $r_{A}$ (see (6)) .

(c) The local results can be used for projection methods such as Arnoldi's method, the generalized minimum residual method(GMREM), the generalized conjugate method(GCM) for combined Newton/finite 
projection methods and in connection to the mesh independence principle in order to develop the cheapest and most efficient mesh refinement strategy [1-3,7,8].

(d) The results can be also be used to solve equations where the operator $F^{\prime}$ satisfies the autonomous differential equation [1-3]:

$$
F^{\prime}(x)=p(F(x)),
$$

where $p$ is a known continuous operator. Since $F^{\prime}\left(x^{*}\right)=p\left(F\left(x^{*}\right)\right)=p(0)$, we can apply the results without actually knowing the solution $x^{*}$. Let as an example $F(x)=e^{x}-1$. Then, we can choose $p(x)=$ $x+1$ and $x^{*}=0$.

(e) It is worth noticing that convergence conditions for method (2) are not changing if we use the new instead of the old conditions [9]. Moreover, for the error bounds in practice we can use the computational order of convergence (COC)

$$
\xi=\frac{\ln \left(\frac{\left\|x_{n+2}-x_{n+1}\right\|}{\left\|x_{n+1}-x_{n}\right\|}\right)}{\ln \left(\frac{\left\|x_{n+1}-x_{n}\right\|}{\left\|x_{n}-x_{n-1}\right\|}\right)}, \quad \text { for each } n=1,2, \ldots
$$

or the approximate computational order of convergence (ACOC)

$$
\xi^{*}=\frac{\ln \left(\frac{\left\|x_{n+2}-x^{*}\right\|}{\left\|x_{n+1}-x^{*}\right\|}\right)}{\ln \left(\frac{\left\|x_{n+1}-x^{*}\right\|}{\left\|x_{n}-x^{*}\right\|}\right)}, \quad \text { for each } n=0,1,2, \ldots,
$$

instead of the error bounds obtained in Theorem 1.

(f) In view of $\left(a_{3}\right)$ and the estimate

$$
\begin{aligned}
\left\|F^{\prime}\left(x^{*}\right)^{-1} F^{\prime}(x)\right\| & =\left\|F^{\prime}\left(x^{*}\right)^{-1}\left(F^{\prime}(x)-F^{\prime}\left(x^{*}\right)\right)+I\right\| \\
& \leq 1+\left\|F^{\prime}\left(x^{*}\right)^{-1}\left(F^{\prime}(x)-F^{\prime}\left(x^{*}\right)\right)\right\| \leq 1+L_{0}\left\|x-x^{*}\right\|,
\end{aligned}
$$

the second condition in $\left(a_{4}\right)$ can be dropped and can be replaced by

$$
v(t)=1+L_{0} t,
$$

or

$$
v(t)=M=2,
$$

since $t \in\left[0, \frac{1}{L_{0}}\right)$.

\section{Semi-local convergence analysis}

Let us modify the (C) conditions ( given in a non-affine invariant form in [9]) so as to be given in affine invariant form as well as introduce the notion of the restricted convergence region. The conditions $(\bar{C})$ are;

Definition 1. The set $T=T\left(F, x_{0}, y_{0}\right)$ belong to class $K=K\left(L_{0}, L, L_{1}, L_{2}, \eta_{0}, \eta\right)$, if

$\left(\bar{C}_{0}\right)\left(\bar{C}_{0}\right)=\left(a_{1}\right)$.

$\left(\bar{C}_{1}\right)$ There exists $x_{0} \in \Omega$ such that $F^{\prime}\left(x_{0}\right)^{-1} \in \mathcal{L}\left(\mathcal{B}_{2}, \mathcal{B}_{1}\right)$.

$\left(\bar{C}_{2}\right)\left\|F^{\prime}\left(x_{0}\right)^{-1}\left(F^{\prime}(x)-F^{\prime}\left(x_{0}\right)\right)\right\| \leq M_{0}\left\|x-x_{0}\right\|$, for each $x \in \Omega$. Set $\Omega_{0}=\Omega \cap B\left(x_{0}, \frac{1}{M_{0}}\right)$, we get $\left\|F^{\prime}\left(x_{0}\right)^{-1} F^{\prime \prime}(x)\right\| \leq \bar{M}$ for each $x \in \Omega_{0}$.

$\left(\bar{C}_{3}\right)\left(\bar{C}_{3}\right)=\left(C_{3}\right)$.

$\left(\bar{C}_{4}\right)\left\|F^{\prime}\left(x_{0}\right)^{-1}\left(F^{\prime \prime}(x)-F^{\prime \prime}(y)\right)\right\| \leq \bar{\varphi}(\|x-y\|)$ for each $x, y \in \Omega_{0}$, where $\bar{\varphi}$ is as $\varphi$.

We shall compare, the old condition $(C)$ assuming they are given in affine invariant form with the new conditions $(\bar{C})$. Clearly,

$$
\begin{gathered}
\Omega_{0} \subseteq \Omega, \\
M_{0} \leq \bar{M} \leq M, \\
\bar{\varphi}(t) \leq \varphi(t),
\end{gathered}
$$


hold in general and $\frac{\bar{M}}{M_{0}}$ can be arbitrarily large [1-3]. With these modifications as in [9], we define the functions

$$
\begin{aligned}
\bar{\varphi}(t)= & \bar{g}_{1}(t)+(1+t) \bar{g}_{2}(t)+(1+t+|\gamma|) \bar{g}_{3}(t) \\
\bar{h}(t)= & \frac{1}{1-\bar{\varphi}(t) t} \\
\bar{\psi}(t, u)= & \left(\frac{2}{3}\right)^{p} \bar{\varphi}_{2}(t, u) u+t^{2}(1+|\gamma|+|\gamma| t) \bar{\varphi}_{2}(t, u)+\frac{1}{p+1}\left(1+t+|\gamma| t^{2}\right) \bar{\varphi}_{2}(t, u) u \\
& +\frac{t^{2}\left(1+t+|\gamma| t^{2}\right)}{1(1-\delta t)} \bar{\varphi}_{2}(t, u)+t(1+t) \bar{\varphi}_{1}(t, u)\left(1+t+|\gamma| t^{2}\right) \bar{\varphi}_{2}(t, u) \\
& +\frac{1}{2}\left(1+t+|\gamma| t^{2}\right)^{2} \bar{\varphi}_{2}(t, u)^{2}
\end{aligned}
$$

where

$$
\begin{aligned}
\bar{g}_{1}(t) & =1+\frac{1}{2(1-\delta t)}, \\
\bar{g}_{2}(t) & =\frac{t}{2}+\frac{t}{2(1-\delta t)}+\frac{t^{2}}{2(1-\delta t)}+\frac{t^{3}}{8(1-\delta t)^{2}}, \\
\bar{g}_{3}(t) & =t \bar{g}_{2}(t)+t(1+t) \bar{g}_{2}(t)+\frac{t^{2}(1+t)}{2(1-\delta t)} \bar{g}_{2}(t)+\frac{t}{2}(1+t)^{2} \bar{g}_{2}^{2}(t), \\
\bar{\varphi}_{1}(t, u) & =\frac{2^{p-1}}{3^{p}} u+\frac{u}{(p+1)(p+2)}+\frac{(1+\delta) t^{2}}{2(1-\delta t)}+\frac{t^{3}}{8(1-\delta t)^{2}}, \\
\bar{\varphi}_{2}(t, u) & =\left[\left(\frac{2}{3}\right)^{p} u+t^{2}+\frac{(1+t) u}{p+1}+\frac{t^{2}(1+t)}{2(1-\delta t)}\right] \bar{\varphi}_{1}(t, u)+\frac{t}{2}(1+t)^{2} \bar{\varphi}_{1}(t, u)^{2} .
\end{aligned}
$$

Moreover, define $\bar{\beta}=1, \eta_{0}=\eta, a_{0}=\bar{M} \eta, b_{0}=\eta \bar{\varphi}(\eta)$ and $C_{0}=h\left(a_{0}\right) \bar{\psi}\left(a_{0}, b_{0}\right)$. Define the sequences

$$
\left\{\begin{array}{l}
\bar{\beta}_{n+1}=\bar{h}\left(a_{n}\right) \bar{\beta}_{n}, \bar{\eta}_{n+1}=\bar{C}_{n} \bar{\eta}_{n,} \\
\bar{a}_{n+1}=\bar{M} \bar{\beta}_{n+1} \bar{\eta}_{n+1}, \bar{b}_{n+1}=\bar{\beta}_{n+1} \bar{\eta}_{n+1} \bar{\varphi}\left(\bar{\eta}_{n+1}\right), \\
\bar{e}_{n+1}=\bar{h}\left(\bar{a}_{n+1}\right) \bar{\psi}\left(\bar{a}_{n+1}, \bar{b}_{n+1}\right) .
\end{array}\right.
$$

Next, we present the semi-local convergence analysis of method (2) using the conditions $(\bar{C})$ instead of conditions $(C)$ used in [9].

Theorem 2. Suppose that the conditions $(\bar{C})$ hold and $\bar{U}\left(x_{0}, \bar{R} \eta\right) \subseteq \Omega$, where $\bar{R}=\frac{\bar{\varphi}\left(\bar{a}_{0}\right)}{1-\bar{c}_{0}}$. For $\bar{a}_{0}=M \eta, b_{0}=$ $\eta \bar{\varphi}(\eta), \bar{c}_{0}=\bar{h}\left(\bar{a}_{0}\right) \bar{\psi}\left(\bar{a}_{0}, \bar{b}_{0}\right)$. Suppose $\bar{a}_{0}<\bar{s}^{*}$ and $\bar{h}\left(\bar{a}_{0}\right) \bar{c}_{0}<1$, where $\bar{s}^{*}$ is the smallest positive solution of equation $\bar{\varphi}(t) t-1=0$. Then, sequence $\left\{x_{n}\right\}$ starting from $x_{0} \in \Omega_{0}$ and generated by method (2) is well defined in $U\left(x_{0}, \bar{R} \eta\right)$, remains in $U\left(x_{0}, \bar{R} \eta\right)$ for each $n=0,1,2, \ldots$ and converges to a unique solution $x^{*} \in \Omega_{1}=\Omega \cap U\left(x_{0}, \tilde{R}\right)$, where $\tilde{R}=\frac{2}{\overline{a_{0}}}-\bar{R}$. Moreover, the following estimates hold;

$$
\left\|x_{n}-x^{*}\right\| \leq e_{n}
$$

where $e_{n}=\bar{\varphi}\left(\bar{a}_{0}\right) \eta \alpha^{n} \varepsilon^{\frac{(4+3 a)^{n}-1}{3+3 a}} \frac{1}{1-\alpha \varepsilon^{(4+3 a)^{n}}}, \quad \alpha=\frac{1}{\bar{h}\left(\bar{a}_{0}\right)}, \quad \varepsilon=\bar{h}\left(\bar{a}_{0}\right) \bar{c}_{0}$.

Proof. Simply notice that iterate belong in $\Omega_{0}$ which is a more precise location than $\Omega$ used in [9]. Hence, the proof of Theorem 1 can be repeated but using the bar constants and functions instead of the non bar constants and functions, which is an important modification (see also the Remark that follows).

Remark 2. In view of (37)-(39), we have the advantages mentioned in the introduction of this study. For example,

$$
\begin{gathered}
a_{0}=M \eta<s^{*} \Longrightarrow \bar{a}_{0}=\bar{M} \eta<\bar{s}^{*}, \\
h\left(a_{0}\right) c_{0}<1 \Longrightarrow \bar{h}\left(\bar{a}_{0}\right) \bar{c}_{0}<1,
\end{gathered}
$$

but not necessarily vice versa, where $s^{*}$ is the smallest positive solution of $\varphi(t) t-1=0$. 


\section{Numerical examples}

We present the following example to test the convergence criteria.

Example 1. Let $\mathbb{B}_{1}=\mathbb{B}_{2}=\mathbb{R}^{3}, D=B(0,1), x^{*}=(0,0,0)^{T}$. Define $F$ on $D$ by

$$
F(u)=F\left(u_{1}, u_{2}, u_{3}\right)=\left(e^{u_{1}}-1, u_{2}^{2}+u_{2}, u_{3}\right)^{T} .
$$

For the points $u=\left(u_{1}, u_{2}, u_{3}\right)^{T}$, the Fréchet derivative is given by

$$
F^{\prime}(u)=\left(\begin{array}{ccc}
e^{u_{1}} & 0 & 0 \\
0 & 2 u_{2}+1 & 0 \\
0 & 0 & 1
\end{array}\right) .
$$

Using the norm of the maximum of the rows and $\left(a_{3}\right)-\left(a_{4}\right)$, we see that since $F^{\prime}\left(x^{*}\right)=\operatorname{diag}(1,1,1)$, we can define functions for method (2) by $w_{0}(t)=(e-1) t, w(t)=e^{\frac{1}{e-1}} t, \quad v(t)=\bar{v}(t)=e^{\frac{1}{e-1}}$. Then, the radius of convergence using (10) is given by

$$
\begin{aligned}
& r_{1}=0.13043162089314655482930049856805, \\
& r_{2}=0.015754168279106986472193341342063=r, \\
& r_{3}=0.064012457484415613562234170785814 .
\end{aligned}
$$

Local results were not given in [9] but if they were, then $w_{0}(t)=w(t)=e t, \bar{v}(t)=v(t)=e$, so old

$$
\begin{aligned}
& r_{1}=0.052167745643160665092175065638003, \\
& r_{2}=0.0017930634000776965713414012881799=r, \\
& r_{3}=0.020054377144156372569927526683387 .
\end{aligned}
$$

\section{Conclusion}

Major concerns in the study of the convergence for iterative methods (local or semilocal) are; the size of the convergence domain, the selection of the initial point and the uniqueness of the solution. We address these problems using method (2) under sufficient convergence conditions which are weaker than the ones in [9] for the semilocal convergence case. This way we extend the convergence domain require fewer iterates to achieve a desired error tolerance and provide a better location on the location of the solution. We also examine the local convergence case not studied in [9]. In the future we will employ our technique to extend the applicability of other iterative methods too.

Author Contributions: All authors contributed equally to the writing of this paper. All authors read and approved the final manuscript.

Conflicts of Interest: "The authors declare no conflict of interest."

\section{References}

[1] Argyros, I. K., \& Magreñán, A. A. (2017). Iterative Methods and Their Dynamics with Applications. CRC Press, New York.

[2] Argyros, I. K., George, S., \& Thapa, N. (2018). Mathematical Modeling for the Solution of Equations and Systems of Equations with Applications, (Volume-I). Nova Publishes, New York.

[3] Argyros, I. K., George, S., \& Thapa, N. (2020). Mathematical Modeling for the Solution of Equations and Systems of Equations with Applications, (Volume-IV). Nova Publishes, New York.

[4] Ezquerro, J. A., \& Hernández, M. A. (2005). On the R-order of the Halley method. Journal of Mathematical Analysis and Applications, 303, 591-601.

[5] Hernández, M. A., \& Salanova, M. A. (2000). Modification of the Kantorovich assumptions for semilocal convergence of the Chebyshev method. Journal of Computational and Applied Mathematics, 126, 131-143.

[6] Hernández, M. A., \& Martinez, E. (2015). On the semilocal convergence of a three step Newton-type iterative process under mild convergence conditions. Numerical Algorithms, 70(2), 377-392.

[7] Magreñán, A. A., \&Argyros, I. K. (2017). Iterative Algorithms II. Nova Publishes, New York.

[8] Singh, M. K., \& Singh, A. K. (2020). Variants of Newton's method using Simpson's 3/8 ${ }^{\text {th }}$ rule. International Journal of Applied and Computational Mathematics, 6, Article No. 20. 
[9] Wang, X., \& Kou, J. (2018). Semilocal convergence for a class of improved multi-step Chebyshev-Halley-like methods under extended conditions. Journal of Fixed Point Theory and Applications, 20, Article No. 122.

(C) 2021 by the authors; licensee PSRP, Lahore, Pakistan. This article is an open access article distributed under the terms and conditions of the Creative Commons Attribution (CC-BY) license (http://creativecommons.org/licenses/by/4.0/). 